\title{
Context-Dependent Olfactory Learning in an Insect
}

\author{
Yukihisa Matsumoto and Makoto Mizunami ${ }^{1}$ \\ Graduate School of Life Sciences, Tohoku University, Aoba-ku, Katahira 2-1-1, Sendai 980-8577, Japan
}

\begin{abstract}
We studied the capability of the cricket Gryllus bimaculatus to select one of a pair of odors and to avoid the other in one context and to do the opposite in another context. One group of crickets was trained to associate one of a pair of odors (conditioned stimulus, CSI) with water reward (appetitive unconditioned stimulus, US+) and another odor (CS2) with saline solution (aversive US, US-) under illumination and to associate CS1 with US- and CS2 with US+ in the dark. Another group of crickets received training of the opposite stimulus arrangement. At $1 \mathrm{~d}$ after the training for $3 \mathrm{~d}$, the former group significantly preferred CS1 over CS2 under illumination but preferred CS2 over CS1 in the dark, and the latter group exhibited the opposite odor preference. The results of control experiments showed that the background light condition had no significant effects on memory formation or retrieval unless it was explicitly associated with US during training. Thus, the visual context affected learning performance only when crickets were requested to use it to disambiguate the meaning of CSs and to predict USs.
\end{abstract}

Animals learn that an originally neutral stimulus can act as a predictor (conditioning stimulus, CS) for a biologically significant stimulus (unconditioned stimulus, US). Such elementary association between CS and US can be acquired by a great variety of animals through classical conditioning. Insects have been used as pertinent models in which to study principles governing elementary associative learning and its underlying molecular and cellular mechanisms. In the fruit fly Drosophila, single gene mutants with defects in the cAMP signaling pathway, such as rutabaga and dunce, are also defective in olfactory learning and memory (Davis 1996; Dubnau and Tully 1998), and evidence suggests that expression of rutabaga-encoded adenylyl cyclase in the mushroom body (MB), a higher olfactory center that integrates multisensory signals (Schildberger 1984; Li and Strausfeld 1997, 1999; Mizunami et al. 1998a; Okada et al. 1999; Strausfeld and Li 1999), is necessary for the formation of olfactory short-term memory (Zars et al. 2000; McGuire et al. 2003).

Conditioning in mammals often depends on the general "context" in which association between the CS and US occurs. In rats, context-dependent fear conditioning in which animals received different CS-US associations under different contexts has been studied (Holland and Bouton 1999; Maren and Holt 2000), and it has been shown that this form of nonelementary associative learning depends crucially on intact hippocampus, but elementary associative learning does not (Hirsh 1974; Holland and Bouton 1999). In insects, the capability of context-dependent learning has been demonstrated for visual pattern discrimination learning (in bumblebees: Colborn et al. 1999; Fauria et al. 2002; in ants: Chameron et al. 1998) and for learning of the position of a visual target (in cockroaches: Kwon et al. 2004). However, no context-dependent form of olfactory learning, which is useful for the study of underlying neural processes, has been established in any insects. We have shown that crickets easily associate olfactory CS with gustatory US and retain olfactory memory for practically a lifetime (Matsumoto and Mizunami 2000, 2002a,b). In this study, we show that crickets have the capability of selecting one of a pair of odors and avoiding the other under one light condition and doing the opposite under another light condition.

\footnotetext{
'Corresponding author.
}

E-MAIL makoto@biology.tohoku.ac.jp; FAX 81-22-217-5050.

Article and publication are at http://www.learnmem.org/cgi/doi/10.1101/ Im.72504.

\section{RESULTS}

Because environmental light conditions may potentially modulate various aspects of innate and learned behaviors of animals, we first studied (1) the effect of light conditions on initial odor preference, (2) the effect of light conditions during training or testing on the level of memory formation or retrieval in elementary associative learning, and (3) the effect of change of light conditions in training and testing on the level of memory retrieval in crickets. The results of these studies provided a basis for the design of experiments to study context-dependent olfactory learning capability in crickets. Training was done by placing individual crickets in a beaker and presenting vanilla odor or peppermint odor before the presentation of water reward or saline (Fig. 1A,B), and the odor preferences of individual crickets were tested in a box by allowing them to freely choose between vanilla and peppermint sources (Fig. 1C,D).

\section{Effect of Background Light Condition on Initial Odor Preference}

In the initial preference test done under illumination, crickets $(n=114)$ in five groups (PT-1 in Fig. 2; PT-0 of groups A and B in Fig. 3; PT-0 of groups A and B in Fig. 4) exhibited significantly greater preference for vanilla odor over peppermint odor: Within-group comparison showed that they spent significantly more time at the vanilla source than at the peppermint source $\left(t_{\mathrm{V}}=17.4 \pm 0.8 \mathrm{sec}, t_{\mathrm{P}}=8.8 \pm 0.8 \mathrm{sec}, n=114, p<0.0001\right.$; $\mathrm{df}=1, T=672.0$, WCX test; means $\pm \mathrm{SEM}$ ). No significantly different initial odor preferences were found among the five groups of crickets used in this study ( $p>0.5, \mathrm{df}=4, H=0.280$, K-W test).

One group of untrained crickets was subjected to odor preference tests in the dark and under illumination (Fig. 2). Crickets exhibited no significantly different odor preferences under different light conditions: Within-group comparison showed that crickets spent significantly more time at the vanilla source than at the peppermint source in tests performed in the dark (PT-2 in Fig. $2 ; t_{\mathrm{V}}=17.5 \pm 3.1 \mathrm{sec}, t_{\mathrm{p}}=6.7 \pm 1.3 \mathrm{sec}, n=26, p<0.001$, $\mathrm{df}=1, T=24.5$, WCX test), and between-group comparison showed that the preference for peppermint in the dark did not significantly differ from that under illumination (PT-1, $p>0.5$, $\mathrm{df}=1, T=118.0$, WCX test). Thus, the background light condition had no significant effect on innate odor preference in crickets. 

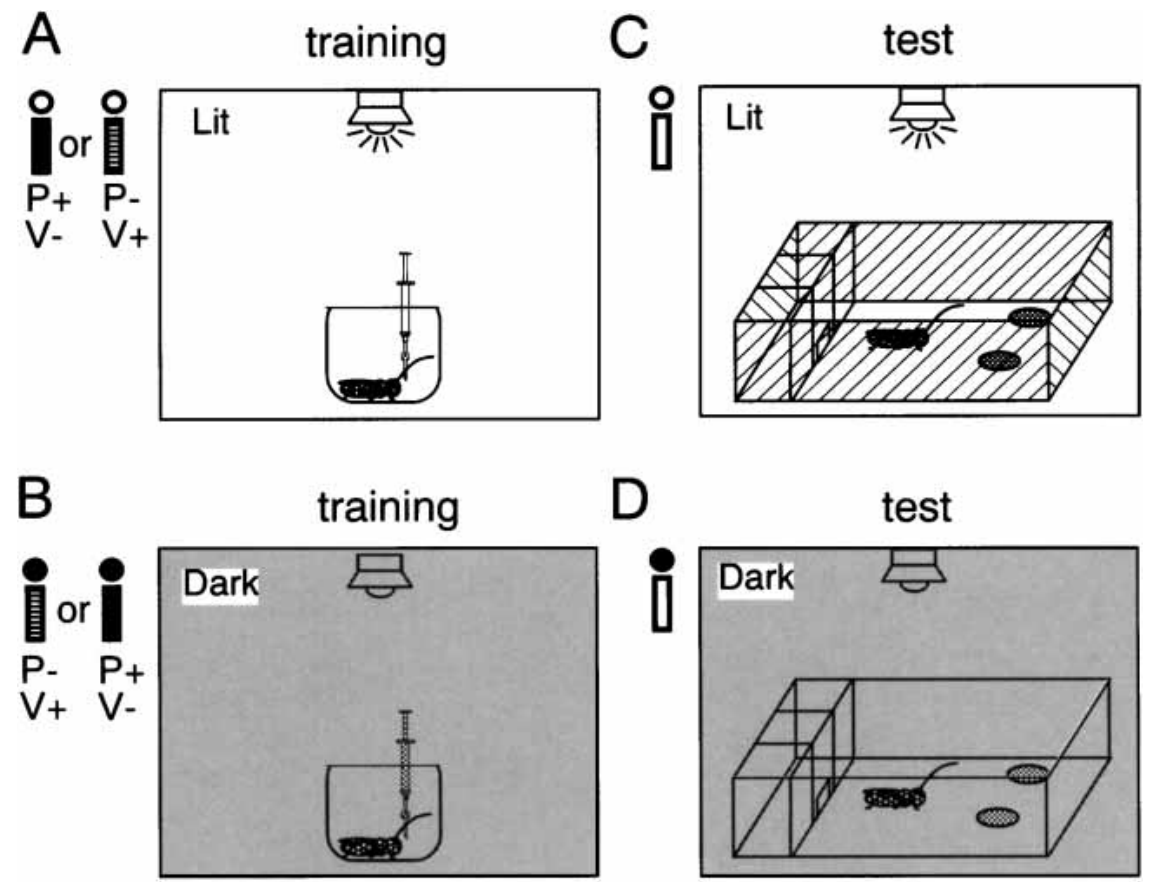

Figure 1 Simplified diagrams of the experimental setup. Crickets were subjected to training or testing in the lit or dark condition. For training $(A, B)$, each cricket was presented with peppermint or vanilla odor before the presentation of water (reward) or saline (non-reward) in a beaker. For odor preference test $(C, D)$, each cricket was allowed to choose freely between peppermint and vanilla sources in a box. For training or testing under illumination $(A, C)$, a white fluorescent lamp on the ceiling was turned on. The black bar indicates training to associate peppermint odor with water and vanilla odor with saline solution ( $\mathrm{P}+$ and $\mathrm{V}-$ conditioning trials), the hatched bar indicates training to associate vanilla odor with water and peppermint odor with saline solution $\left(\mathrm{P}-\right.$ and $\mathrm{V}_{+}$ conditioning trials), and the white bar indicates odor preference test. The white and black circles above the bars indicate lit and dark conditions, respectively.

\section{Effect of Background Light Condition on Elementary Associative Learning}

To determine whether or not the background light condition during training or testing affects memory acquisition or retrieval in elementary associative learning, two groups of crickets were subjected to initial odor preference tests, and then one group (group A) was subjected to training under illumination and another group (group B) was subjected to training in the dark (Fig. 3). At $1 \mathrm{~d}$ after training, their odor preferences were tested both under illumination and in the dark. Training consisted of two sessions of differential conditioning trials to associate peppermint with water $(\mathrm{P}+$ conditioning) and to associate vanilla with saline solution ( $\mathrm{V}$ - conditioning) with a time interval between sessions of $2 \mathrm{~h}$, one training session consisting of four conditioning trials with the sequence of $\mathrm{P}+, \mathrm{V}-, \mathrm{P}+$, and $\mathrm{V}-$ conditioning trials.

After conditioning, crickets in group A exhibited significantly greater preferences for peppermint (rewarded odor) over vanilla (negatively reinforced odor) under illumination and in the dark: They spent significantly more time at the peppermint source than at the vanilla source both under illumination and in the dark $\left(t_{\mathrm{P}}=23.2 \pm 3.7 \mathrm{sec}, t_{\mathrm{V}}=6.5 \pm 1.5 \mathrm{sec}, n=17, p<0.001\right.$, $\mathrm{df}=1, T=0.0$ in PT $-1 ; t_{\mathrm{P}}=22.1 \pm 3.6 \mathrm{sec}, t_{\mathrm{V}}=5.9 \pm 0.6 \mathrm{sec}$, $n=17, p<0.001, \mathrm{df}=1, T=0.0$ in PT-2, WCX test). The preference for peppermint in tests conducted under illumination was significantly greater after training (PT-1) than before training (PT-0; $p<0.001, \mathrm{df}=1, T=1.0$, WCX test) and that the preference for peppermint in tests conducted in the dark was significantly greater in the trained group (PT-2) than in the untrained group (PT-2 in Fig. 1 ; $p<0.0001, \mathrm{df}=1, U=18.5$, M-W test).

Similar results were obtained in group B. After conditioning, crickets in group B exhibited significantly greater preferences for peppermint over vanilla under illumination and in the dark: They spent significantly more time at the peppermint source than at the vanilla source both in the dark and under illumination $\left(t_{\mathrm{P}}=19.6 \pm 3.5 \mathrm{sec}, t_{\mathrm{V}}=10.6 \pm 2.6\right.$ sec, $n=17, p<0.05, \mathrm{df}=1, T=27.5$ in PT$1 ; t_{\mathrm{P}}=21.4 \pm 2.2 \mathrm{sec}, t_{\mathrm{V}}=8.6 \pm 1.0 \mathrm{sec}$, $n=17, p<0.001, \mathrm{df}=1, T=4.0$ in PT -2 , WCX test). In addition, the preference for peppermint in tests conducted under illumination was significantly greater after training (PT-2) than before training (PT-0) ( $p<0.001, \mathrm{df}=1, T=5.0$, WCX test $)$, and the preference for peppermint in tests conducted in the dark was significantly greater in the trained group (PT-1) than in the untrained group (PT-2 in Fig. 2; $p<0.0001$, $\mathrm{df}=1, U=47.0, \mathrm{M}-\mathrm{W}$ test).

The results of the Kruskal-Wallis test showed that the preferences for peppermint under illumination and in the dark in groups that had undergone training under illumination (group A) and in the dark (group B; Fig. 3) were not significantly different $(p>0.05, \mathrm{df}=3, H=2.953, \mathrm{~K}-\mathrm{W}$ test). This indicates that (1) background light condition during training had no significant effect on the level of memory formation: The preference for peppermint of crickets that had undergone training under illumination (PT-1 and PT-2 in group A) did not significantly differ from that of crickets that had undergone training in the dark (PT-1 and PT-2 in group B) regardless of light conditions during testing; (2) the light condition during testing had no significant effect on the level of memory retrieval: The preference for peppermint tested under illumination (PT-1 in group A and PT-2 in group B) did not significantly differ from that tested in the dark (PT-2 in group B and PT-1 in group B) regardless of the light condition during training; and (3) the change in the light condition in training and testing had no significant effect on the level of memory retrieval: The preference for peppermint tested in a condition of illumination different from that used in training (PT-2 in group A and PT-1 in group B) did not significantly differ from that tested in conditions of illumination similar to that used in training (PT-1 in group A and PT-2 in group B). Thus, visual context did not significantly affect olfactory memory formation or retrieval in elementary associative learning. This finding allowed for use of a simple paradigm to study the context-dependent olfactory learning capability of crickets.

\section{Effect of Context-Dependent Olfactory Conditioning Trials}

In the experiment for which the results are shown in Figure 4, crickets in groups A and B were subjected to initial odor preference tests under illumination (PT-0). Then crickets in group A were subjected to $\mathrm{P}+$ and $\mathrm{V}-$ conditioning trials under illumination and $\mathrm{P}-$ and $\mathrm{V}+$ conditioning trials in the dark, and crickets in group $\mathrm{B}$ were subjected to $\mathrm{P}-$ and $\mathrm{V}+$ conditioning trials under illumination and $\mathrm{P}+$ and $\mathrm{V}-$ conditioning trials in the dark. Crickets in group A and group B were subjected to two training 

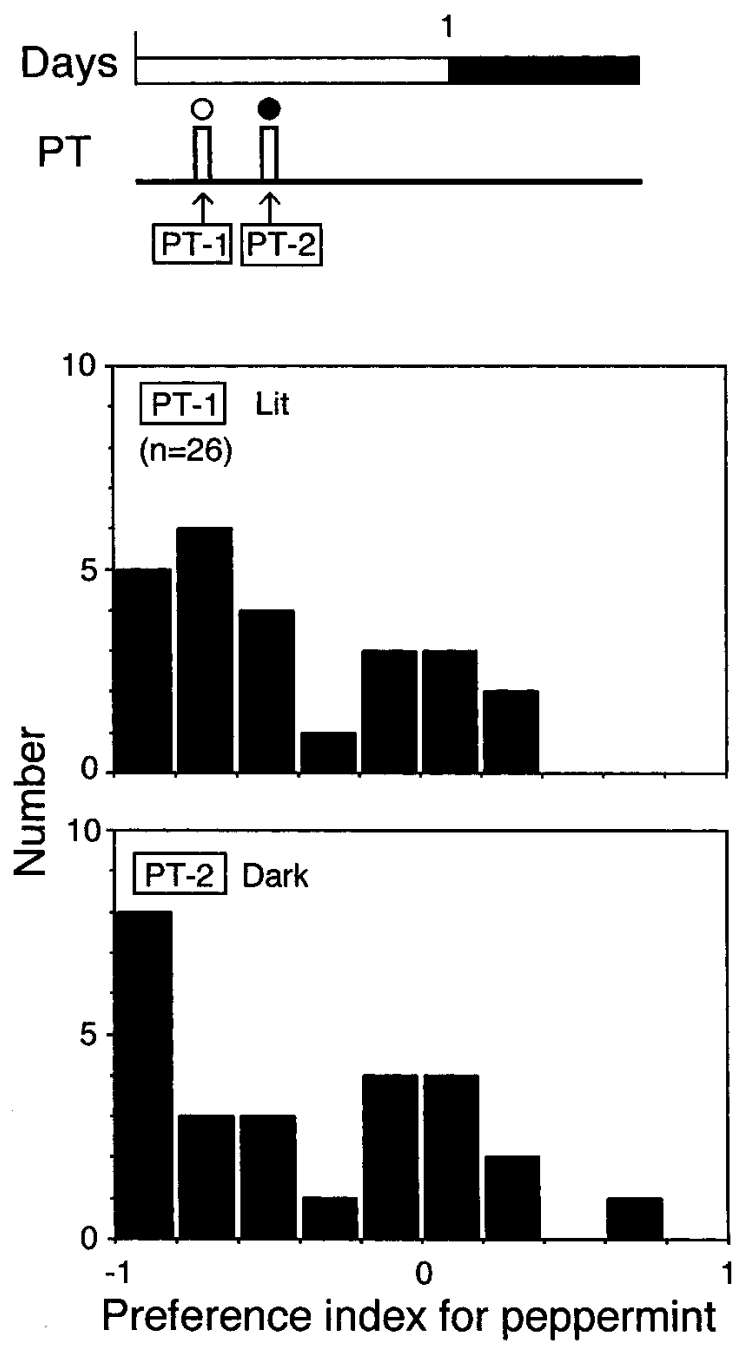

Figure 2 Odor preference of untrained crickets tested under illumination (upper) and in the dark (lower). The diagram at the top shows the time schedule for the preference test (PT, white bars). The white and black circles above the bars indicate lit and dark conditions, respectively. The white and black parts of the time bar indicate photophase $(12 \mathrm{~h})$ and scotophase $(12 \mathrm{~h})$, respectively. Histograms show the distributions of preference index $(\mathrm{PI})$ for peppermint for each individual of a group of crickets tested under illumination (PT-1) and in the dark (PT-2). The number $(n)$ indicates sample size.

sessions with a time interval between sessions of $1.5 \mathrm{~h}$ under illumination or in the dark and then two more training sessions in the dark or under illumination on each of three consecutive days. Each training session consisted of four conditioning trials (e.g., $\mathrm{P}+, \mathrm{V}-, \mathrm{P}+$, and $\mathrm{V}-$ conditioning trials).

At $1 \mathrm{~d}$ after training, crickets in group A exhibited a significantly greater preference for peppermint over vanilla under illumination (PT-1; within-group comparison: $t_{\mathrm{P}}=23.0 \pm 3.1 \mathrm{sec}$, $t_{\mathrm{V}}=10.5 \pm 1.5 \mathrm{sec}, n=29, p<0.005, \mathrm{df}=1, T=61.0$, WCX test) but exhibited significantly greater preference for vanilla over peppermint in the dark $\left(\mathrm{PT}-2 ; t_{\mathrm{V}}=29.7 \pm 3.8 \mathrm{sec}, t_{\mathrm{P}}=9.9 \pm 1.6\right.$ sec, $n=29, p<0.0001$, df $=1, T=1.0$, WCX test). After conditioning, the odor preferences of crickets differed significantly under different light conditions: The preference for peppermint tested under illumination (PT-1) was significantly greater than that tested in the dark (PT-2; $p<0.0001, \mathrm{df}=1, T=10.0$, WCX test).

Similarly, crickets in group B exhibited a significantly greater preference for vanilla over peppermint under illumination $\left(\mathrm{PT}-1 ; t_{\mathrm{V}}=23.8 \pm 2.1 \mathrm{sec}, t_{\mathrm{P}}=11.8 \pm 1.8 \mathrm{sec}, n=25\right.$, $p<0.0001$, df $=1, T=4.0$, WCX test) but exhibited a significantly greater preference for peppermint over vanilla in the dark $\left(\mathrm{PT}-2 ; t_{\mathrm{P}}=25.8 \pm 1.5 \mathrm{sec}, t_{\mathrm{V}}=10.2 \pm 1.5 \mathrm{sec}, n=25, p<0.0001\right.$, $\mathrm{df}=1, T=9.0$, WCX test). After conditioning, the preference for peppermint under illumination (PT-1) was significantly less than that in the dark (PT-2; $p<0.0001, \mathrm{df}=1, T=4.0$, WCX test). A comparison of groups A and B showed that odor preferences of these two groups significantly differed in tests conducted under illumination (PT-1: $p<0.0001, \mathrm{df}=1, U=70.0, \mathrm{M}-\mathrm{W}$ test) and in the dark (PT-2: $p<0.0001, \mathrm{df}=1, U=22.0, \mathrm{M}-\mathrm{W}$ test), reflecting different stimulus arrangements during training in the two groups. We conclude that crickets learn to select one of a pair of odors and to avoid the other in one context and do the opposite in a different context.

\section{DISCUSSION}

Although environmental light conditions may modulate many aspects of innate and learned behaviors of animals, we found no effects of background light condition on innate odor preference or on performance after elementary associative learning in crickets. For the latter, the light conditions during training or testing had no significant effects on the levels of memory formation or retrieval, and the change of light condition in training and testing had no significant effect on the level of memory retrieval. In relation to this observation, it is worth noting that our training was carried out in a classical conditioning situation in which crickets were sequentially subjected to CS1-US+ and CS2-USpairing trials in a beaker, and then their odor preference was tested in an operant situation in which two CSs were presented simultaneously in an arena so that crickets could freely choose between them. Nevertheless, the training effects could be transferred from the classical conditioning situation to the operant test situation. The results show that context generalization, rather than context specificity, guides elementary associative learning in crickets.

We showed that crickets are capable of learning to select one of a pair of odors and avoid the other in one condition of illumination and to do the opposite in another condition. A similar context-dependent form of conditioning has been studied extensively in rats, and two theories have been proposed to account for this form of conditioning (Holland and Bouton 1999; Pearce and Bouton 2001). One explanation (occasion-setting theory) is that the animal uses the contextual stimulus to "set the occasion" for explicit relations between CSs and USs, that is, to use context signals to disambiguate CS and to drive an appropriate conditioned response. In the other theory (configural theory), the animal configures explicit CSs and background contextual stimulus and perceives different CS-context combinations as different stimuli, each of which would then be separately associated with each respective US. Context-dependent visual pattern discrimination learning capability has been demonstrated in bumblebees, and it has been argued that either the former theory (Colborn et al. 1999) or the latter theory (Fauria et al. 2002) may account for this capability. It would be interesting to study which of the two theories better explains contextual olfactory learning in crickets. The capability of insects to form nonelemental, configural association has been demonstrated in honey bees: They form configural association between two distinct odors in discriminatory olfactory learning (Deisig et al. 2001, 2002).

The exact nature of visual stimuli that crickets used for discriminating the lit condition from dark condition remains to be determined. Because the training was made in a transparent beaker and the test was made in a box made of black Lucite plates, 


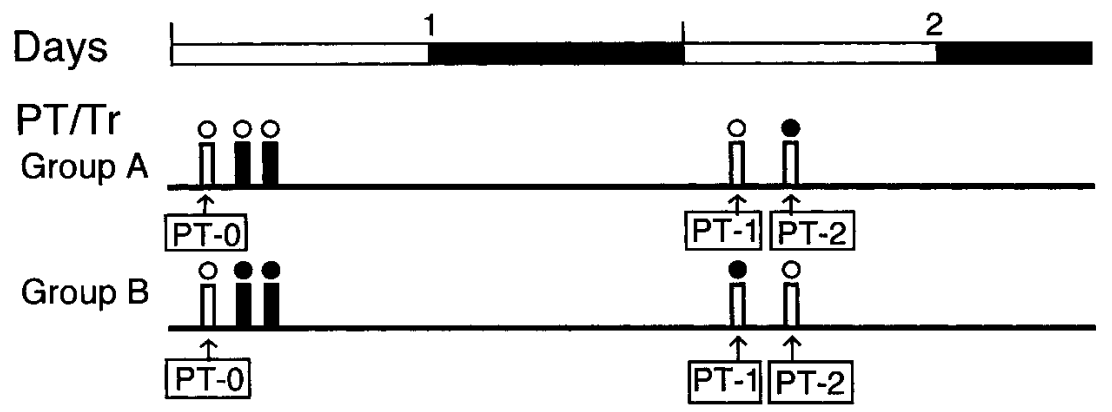

Group A

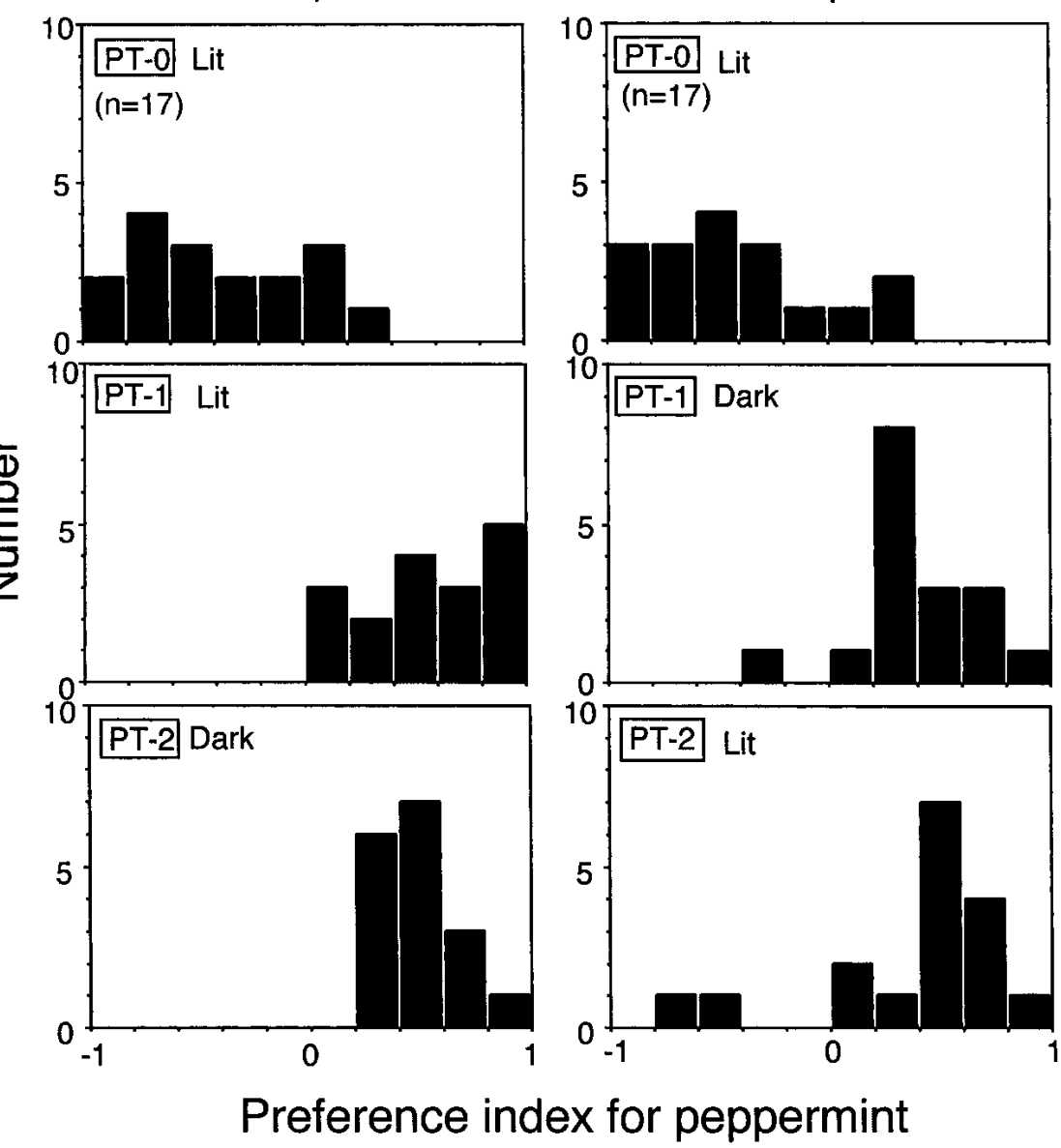

Figure 3 Effects of background light condition on elementary associative learning. The diagram at the top shows the time schedules for the preference test (PT, white bars) and training ( $\mathrm{Tr}$, black bars). The white and black circles above the bars indicate lit and dark conditions, respectively. One group of crickets was subjected to training under illumination (group A), and the other group was subjected to training in the dark (group $B$ ). Upper histograms show the distributions of preference index $(\mathrm{PI})$ for peppermint for each individual of two groups of crickets tested under illumination before training (PT-0); middle and lower histograms show those tested under illumination (PT-1 in group A and PT2 in group B) and those tested in the dark (PT-2 in group A and PT-1 in group B) $1 \mathrm{~d}$ after training. The number $(n)$ indicates sample size.

the visual scene crickets experienced under illumination in the training and testing situations differed in large part (see Fig. $1 \mathrm{~A}, \mathrm{C})$. Thus, it is less likely that crickets used particular visual objects to define the lit condition. Either the lightness or the presence of visual scene or both may have been used to define the lit condition.

An important question to be addressed in our ongoing studies is the areas of the insect brain in which the olfactory CS pathway, gustatory US pathway, and visual contextual stimulus pathway converge to form context-dependent olfactory memory. Studies in honeybees suggest that the olfactory CS pathway and gustatory US pathway converge in three areas of the brain, namely, the antennal lobe (AL), calyx of the mushroom body (MB), and the lateral protocerebrum in the case of elementary associative learning (Hammer 1993). Among these three areas, it seems unlikely that the $\mathrm{AL}$ is the site in which context-dependent olfactory memory is formed because this primary olfactory center receives few visual signals. The MB is known to participate in the processing of contextual signals. Surgical MB ablation in the cockroach resulted in a defect in orientation behavior toward a goal on the basis of visual place memory but not on the basis of direct visual cues (Mizunami et al. 1998b), and in Drosophila, defects in the function of the MB resulted in defects in context generalization in visual pattern discrimination learning (Liu et al. 1999). A multisensory nature of the response of $\mathrm{MB}$ efferent (output) neurons has been noted in crickets (Schildberger 1984) and cockroaches (Li and Strausfeld 1997, 1999; Mizunami et al. 1998a; Okada et al. 1999), and some efferent neurons of the MB even exhibited multisensory conditioned responses; that is, the responses to stimuli of a particular sensory modality were altered depending on the presence or absence of stimuli of another sensory modality (Li and Strausfeld 1999). Many efferent neurons of the $\mathrm{MB}$ project to the lateral protocerebrum (Li and Strausfeld 1997, 1999), and it needs to be studied whether the MB is the area where context-dependent olfactory memory is formed or is a preprocessor of contextual signals, as has been argued by $\mathrm{Li}$ and Strausfeld (1999), and context-specific olfactory memory is formed in the lateral protocerebrum, by integrating contextual signals relayed from the MB with olfactory CS signals and gustatory US signals.

\section{MATERIALS AND METHODS}

\section{Insects}

Adult male crickets, Gryllus bimaculatus de Geer, reared in a 12-h:12-h light:dark photoperiod (photophase: 08:00-20:00) at $27^{\circ} \pm 2^{\circ} \mathrm{C}$, were fed a diet of insect pellets and water ad libitum. Then, $4 \mathrm{~d}$ before the start of the experiment, a group of $\sim 30$ crickets was placed in a container and fed a diet of insect pellets ad libitum; these crickets were deprived of drinking water to enhance their motivation to search for water. On the day of the experiment, they were placed individually in $100-\mathrm{mL}$ glass beakers. All experiments were carried out $1 \mathrm{wk}$ after the imaginal molt.

\section{Olfactory Conditioning and Odor Preference Test}

Figure 1 shows simplified diagrams of the experimental setup for training and testing. A classical conditioning procedure de- 
scribed in our previous paper (Matsumoto and Mizunami 2002a) was used to train crickets to associate one odor (a conditioning stimulus, CS1) with water reward (appetitive conditioned stimulus, US+) and another odor (CS2) with 20\% sodium chloride (aversive US, US -). Artificial essences of peppermint and vanilla were used as CSs. Hypodermic syringes (each $1 \mathrm{~mL}$ ) were used to present CS and US to the animals. A small filter paper was attached to the needle of the syringe $10 \mathrm{~mm}$ from its tip. The syringe used for appetitive conditioning was filled with water, and the filter paper attached to the needle was soaked with one odorant solution. The syringe used for aversive conditioning was filled with saline solution, and the filter paper was soaked with another odorant solution. For the conditioning trial, the filter paper was placed within $1 \mathrm{~cm}$ of the cricket's head, and after 2 sec from the onset of odor presentation, a drop of water or saline solution was placed in the mouth of the cricket for 2 sec. The air in the beaker was then ventilated for $2 \mathrm{sec}$. One training session consisted of four conditioning trials in the sequence appetitive, aversive, appetitive, and then aversive conditioning trials. The intertrial interval was 5 min. After training, each cricket was given a diet of insect pellets ad libitum in a beaker until it was subjected to the odor preference test.

The apparatus used for the odor preference test (Fig. 1C,D) was slightly modified from that used previously (Matsumoto and Mizunami 2002a; Matsumoto et al. 2003). In short, the apparatus was made of black Lucite plates and consisted of three chambers, a "test chamber" and two removable "waiting chambers," one of which was placed at the "waiting position" and the other at the "entrance position." There was a sliding door between the waiting chamber at the entrance position and the test chamber. On the floor of the test chamber, there were two circular holes that connected the chamber with two of three sources of odor. Each odor source consisted of a cylindrical plastic container covered with fine gauze net. The three containers were mounted on a rotatable holder (not illustrated in Fig. 1C,D). Two odor sources could be located simultaneously just below the holes at the "offer position" by rotating the holder.

Before the preference test, a cricket was transferred from the beaker to the waiting chamber at the waiting position and left for $\sim 4$ min to become accustomed to the surroundings. The waiting chamber was then slid into the entrance position, and the door to the test chamber was opened. When the cricket entered the test chamber, the door was closed and the test started. Then, 2 min later, the relative positions of the vanilla and peppermint sources were changed by rotating the container holder. An odor source was considered to have been visited when the cricket probed the top net with its mouth. The time spent visiting each odor source was measured cumulatively. The preference test lasted for 4 min. If the total time of visits of a cricket to either source was $<15 \mathrm{sec}$, we considered that the cricket was less motivated to visit odor sources, possibly because of a ( $n$ ) indicates sample size. bad physical condition, and the data were rejected $(-5 \%$ of crickets fell into this category). At the end of training, the sliding door was opened, and the cricket was gently pushed into the waiting chamber and was then transferred to a beaker. Daily testing and training began at 10:00 $\mathrm{h}$ and lasted a maximum of $6 \mathrm{~h}$. Following testing, crickets were provided a diet of insect pellets ad libitum until the next retention test was given.
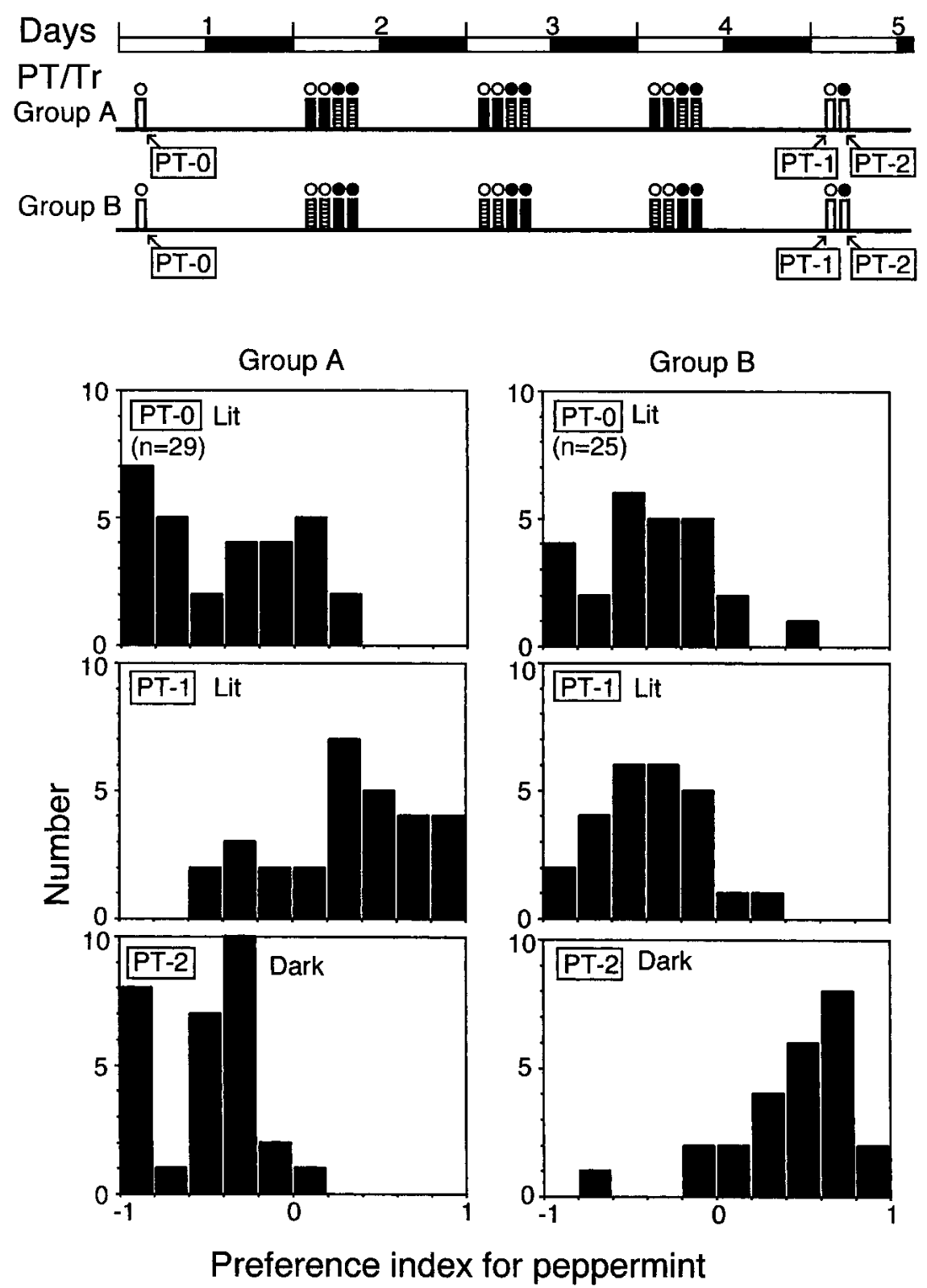

Figure 4 Effects of context-dependent olfactory conditioning trials. The diagram at the top shows the time schedules for the preference test (PT, white bars) and training (Tr, shaded and black bars). The shaded bars indicate training to associate vanilla with water and peppermint with saline solution ( $\mathrm{P}-$ and $\mathrm{V}+$ conditioning trials), and the black bars indicate training to associate peppermint with water and vanilla with saline solution ( $P+$ and $V-$ conditioning trials). One group of crickets (group $\mathrm{A}$ ) was subjected to $\mathrm{P}+$ and $\mathrm{V}-$ conditioning trials under illumination and $\mathrm{P}-$ and $\mathrm{V}_{+}$conditioning trials in the dark, and another group (group $\mathrm{B}$ ) was subjected to $\mathrm{P}-$ and $\mathrm{V}_{+}$ conditioning trials under illumination and $\mathrm{P}+$ and $\mathrm{V}-$ conditioning trials in the dark. Upper histograms show the distributions of preference index for peppermint for each individual in tests conducted before training in a lit condition (PT-0); middle and lower histograms show those tested under illumination (PT-1) and those tested in the dark (PT-2) $1 \mathrm{~d}$ after training for $3 \mathrm{~d}$. The number

\section{Learning \& Memory}

www.learnmem.org 


\section{Background Light Condition}

Crickets were subjected to training or testing under illumination or in the dark. For illuminating the experimental room, a white fluorescent lamp was turned on, the luminance for the test chamber being $\sim 300 \mathrm{~lx}$. Crickets' performance in the dark was observed under dim red light, to which the visual system of crickets appears to be almost insensitive (Lall and Trough 1989; Zufall et al. 1989). For training or testing in the dark, the light was turned off $10 \mathrm{~min}$ before the start of the training or testing session and then turned on $5 \mathrm{~min}$ after the end of the training or testing session.

\section{Data Analysis}

Relative odor preference of crickets in a test was evaluated by comparing the time each cricket spent at the peppermint odor source $\left(t_{\mathrm{P}}\right)$ with that spent at the vanilla odor source $\left(t_{\mathrm{v}}\right)$. Because the distribution of the time did not fit to normal distribution, a nonparametrical Wilcoxon's (WCX) test was used for statistical evaluation. Odor preferences in different tests carried out on the same or different groups of crickets were compared by using the "preference index (PI) for peppermint odor" defined as $\left(t_{\mathrm{p}}-t_{\mathrm{V}}\right) /$ $\left(t_{\mathrm{P}}+t_{\mathrm{V}}\right)$, where PI $>0$ or PI $<0$ indicates that the crickets spent more time at the peppermint odor source than at the vanilla odor source or vice versa. Because the distribution of PIs often deviated from normal distribution, we used nonparametrical tests for statistical evaluation: Wilcoxon's test (WCX) was used to compare the odor preferences in different tests for a given cricket group, and the Mann-Whitney U-test (M-W test) was used for comparison between two cricket groups. The Kruskal-Wallis test (K-W) was used for comparison of the odor preferences in three or more tests. $P$ values of $<0.05$ were considered as statistically significant.

\section{ACKNOWLEDGMENTS}

We thank M. Sakura for helpful discussion and M. Ohara for assistance with language. This study was supported by grant-inaids from the Ministry of Education, Science, Technology, Sports and Culture of Japan (to M.M.) and from the Japan Society for Promotion of Science (to Y.M.).

The publication costs of this article were defrayed in part by payment of page charges. This article must therefore be hereby marked "advertisement" in accordance with 18 USC section 1734 solely to indicate this fact.

\section{REFERENCES}

Chameron, S., Schats, B., Pastergue-Ruiz, I., Beugnon, G., and Collett, T.S. 1998. The learning of a sequence of visual patterns by the ant Cataglyphis cursor. Proc. R. Soc. Lond. B 65: 2309-2313.

Colborn, M., Ahmad-Annuar, A., Fauria, K., and Collett, T.S. 1999. Contextual modulation of visuomotor associations in bumble-bees (Bombus terrestris). Proc. R. Soc. Lond. B 266: 2413-2418.

Davis, R.L. 1996. Physiology and biochemistry of Drosophila learning mutants. Physiol. Rev. 76: 299-317.

Deisig, N., Lachnit, H., Giurfa, M., and Hellstern, F. 2001. Configural olfactory learning in honeybees: Negative and positive patterning discrimination. Learn. Mem. 8: 70-78.

Deisig, N., Lachnit, H., and Giurfa, M. 2002. The effect of similarity between elemental stimuli and compounds in olfactory patterning discriminations. Learn. Mem. 9: 112-121.
Dubnau, J. and Tully, T. 1998. Gene discovery in Drosophila: New insights for learning and memory. Annu. Rev. Neurosci. 21: 407-444.

Fauria, K., Dale, K., Colborn, M., and Collett, T.S. 2002. Learning speed and contextual isolation in bumblebees. J. Exp. Biol. 205: $1009-1018$.

Hammer, M. 1993. An identified neuron mediates the unconditioned stimulus in associative olfactory learning in honeybees. Nature 366: 59-63.

Hirsh, R. 1974. The hippocampus and contextual retrieval of information from memory: A theory. Behav. Biol. 12: 421-444.

Holland, P.C. and Bouton, M.E. 1999. Hippocampus and context in classical conditioning. Curr. Opinion Neurobiol. 9: 195-202.

Kwon, H.-W., Lent, D.D., and Strausfeld, N.J. 2004. Spatial learning in the restrained American cockroach Periplaneta americana. J. Exp. Biol. 207: $377-383$.

Lall, A.B. and Trough, C.D. 1989. The spectral sensitivity of the ocellar system in the cricket Gryllus firmus (Orthoptera: Gryllidae). J. Insect Physiol. 35: 805-808.

Li, Y.-S. and Strausfeld, N.J. 1997. Morphology and sensory modality of mushroom body efferent neurons in the brain of the cockroach, Periplaneta americana. J. Comp. Neurol. 387: 631-650.

. 1999. Multimodal efferent and recurrent neurons in the medial lobes of cockroach mushroom bodies. J. Comp. Neurol. 409: 647-663.

Liu, L., Wolf, R., Ernst, R., and Heisnberg, M. 1999. Context generalization in Drosophila visual learning requires the mushroom bodies. Nature 400: 753-756.

Maren, S. and Holt, W. 2000. The hippocampus and contextual memory retrieval in Pavlovian conditioning. Behav. Brain Res. 110: 97-108.

Matsumoto, Y. and Mizunami, M. 2000. Olfactory learning in the cricket Gryllus bimaculatus. J. Exp. Biol. 203: 2581-2588. . 2002a. Temporal determinants of olfactory long-term retention in the cricket Gryllus bimaculatus. J. Exp. Biol. 205: 1429-1437. . 2002b. Lifetime olfactory memory in the cricket Gryllus bimaculatus. J. Comp. Physiol. A 188: 295-299.

Matsumoto, Y., Noji, S., and Mizunami, M. 2003. Time course of protein synthesis- dependent phase of olfactory memory in the cricket Gryllus bimaculatus. Zool. Sci. 20: 409-416.

McGuire, S.E., Phuong, T.L., Osborn, A.J., Kitamoto, K., and Davis, R.L. 2003. Spatiotemporal rescue of memory dysfunction in Drosophila. Science 302: 1765-1768.

Mizunami, M., Okada, R., Li, Y., and Strausfeld, N.J. 1998a. Mushroom bodies of the cockroach: The activity and identities of neurons recorded in freely moving animals. J. Comp. Neurol. 402: 501-519.

Mizunami, M., Weibrecht, J.M., and Strausfeld, N.J. 1998b. Mushroom bodies of the cockroach: Their participation in place memory. $J$. Comp. Neurol. 402: 520-537.

Okada, R., Ikeda, J., and Mizunami, M. 1999. Sensory responses and movement-related activities in extrinsic neurons of the cockroach mushroom bodies. J. Comp. Physiol. A 185: 115-129.

Pearce, J.M. and Bouton, M.E. 2001. Theories of associative learning in animals. Annu Rev. Psychol. 52: 111-139.

Schildberger, K. 1984. Multimodal interneurons in the cricket brain: Properties of identified extrinsic mushroom body cells. J. Comp. Physiol. A 154: 71-79.

Strausfeld, N.J. and Li, Y.-S. 1999. Organization of olfactory and multimodal afferent neurons supplying the calyx and pedunculus of the cockroach mushroom bodies. J. Comp. Neurol. 409: 603-625.

Zars, T., Fischer, M., Schulz, R., and Heisenberg, M. 2000. Localization of a short-term memory in Drosophila. Science 288: 672-675.

Zufall, F., Schmitt, M., and Menzel, R. 1989. Spectral and polarized light sensitivity of photoreceptors in the compound eye of the cricket (Gryllus bimaculatus). J. Comp. Physiol. A 164: 597-608.

Received November 19, 2003; accepted in revised form March 16, 2004. 


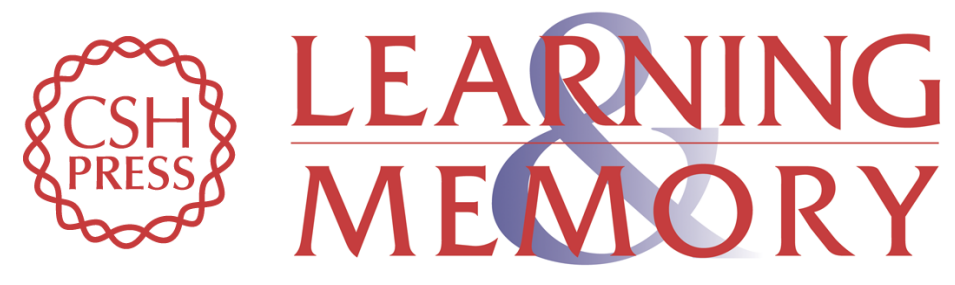

\section{Context-Dependent Olfactory Learning in an Insect}

Yukihisa Matsumoto and Makoto Mizunami

Learn. Mem. 2004, 11:

Access the most recent version at doi:10.1101/lm.72504

References This article cites 29 articles, 8 of which can be accessed free at: http://learnmem.cshlp.org/content/11/3/288.full.html\#ref-list-1

License

Email Alerting Receive free email alerts when new articles cite this article - sign up in the box at the Service top right corner of the article or click here. 